\title{
Transiently Elevated TC and sdLDL-C Levels and Falsely Low LDL-C Levels in Patients with Extrahepatic Cholangiocarcinoma
}

This article was published in the following Dove Press journal: OncoTargets and Therapy

\section{Yi Guo \\ Yong-Gen Zhang \\ Hong-Chun Li \\ Yin-Hai $\mathrm{Xu}$}

Department of Laboratory Medicine, Affiliated Hospital of Xuzhou Medical University, Xuzhou, Jiangsu, People's Republic of China
Correspondence: Yin-Hai Xu Department of Laboratory Medicine, Affiliated Hospital of Xuzhou Medical University, No. 99 West Huaihai Road, Xuzhou, Jiangsu, 221002, People's

Republic of China

Tel +8618705201 I89

Email xyfyxyh@126.com
Purpose: Most patients diagnosed with extrahepatic cholangiocarcinoma (ECCA) exhibit cholestasis caused by obstruction of the bile duct. Cholestasis is associated with lipid disorders, but studies focused on the changing lipid parameters in patients with ECCA are lacking. Here, we observed lipid profiles in patients with ECCA and investigated whether the removal of biliary obstruction could correct dyslipidemia.

Patients and Methods: We consecutively included patients admitted to the hepatobiliary surgery department at the Affiliated Hospital of Xuzhou Medical University. The patients were divided into an ECCA group or a non-ECCA group based on the disease assessment. Patients with histological confirmation of ECCA were included in the ECCA group. Blood samples were collected on admission as well as five days after treatment. An automatic biochemistry analyzer was used to test liver function and serum lipid levels. Serum lipoprotein electrophoresis was performed using barbitone sodium buffer and Sudan black B

Results: A total of 180 patients met inclusion criteria and were enrolled for this study. Of these, 76 patients were diagnosed with ECCA; all other patients were enrolled in the nonECCA group. Total cholesterol (TC) and small and dense low-density lipoprotein cholesterol (sdLDL-C) levels were significantly elevated in the ECCA group. LDL-C levels were found to be slightly lower in the ECCA group. In the ECCA group, serum samples were detained in sample wells and lipoproteins failed to be separated. TC and sdLDL-C levels significantly decreased after cholestasis relief in the ECCA group. Lipoprotein electrophoresis revealed that patients with ECCA showed normal lipoprotein patterns after treatment.

Conclusion: Patients with ECCA exhibited transiently elevated TC and sdLDL-C levels and falsely low LDL-C results. TC, sdLDL-C, and LDL-C levels could be restored to normal levels after biliary obstruction removal and cholestasis relief.

Keywords: extrahepatic cholangiocarcinoma, cholestasis, dyslipidemia, TC, LDL-C, sdLDL-C

\section{Plain Language Summary}

Serum lipid tests at the department of laboratory medicine include total cholesterol (TC), triglycerides (TG), high-density lipoprotein cholesterol (HDL-C), low-density lipoprotein cholesterol (LDL-C), apolipoprotein A (apoA), apolipoprotein B (apoB), lipoprotein (a) (Lp (a)), and small and dense LDL-C (sdLDL-C). Generally, TC values are positively correlated with LDL-C values. In addition, LDL-C values should be greater than sdLDL-C values. However, we recently noticed a patient with extrahepatic cholangiocarcinoma (ECCA) who exhibited biliary obstruction and cholestasis, showed exceptionally high levels of TC $(17.14 \mathrm{mmol} / \mathrm{L})$ and sdLDLC (15.262 mmol/L), and abnormally low levels of LDL-C $(0.48 \mathrm{mmol} / \mathrm{L})$. Clinicians in the 
Department of Hepatobiliary Surgery asked whether they should prescribe the patient lipid-lowering medicines after reviewing these diagnostic results. However, no previous systematic research has been performed that surveyed the changes in lipid parameters in patients with ECCA. Therefore, this study was designed to explore changes in lipid profiles in patients with ECCA and to investigate whether biliary obstruction and cholestasis relief could correct abnormal serum lipid levels. Our data showed that patients with ECCA had transiently elevated levels of TC and sdLDL-C and falsely low LDL-C results. TC, sdLDL-C, and LDL-C levels could be recovered to normal levels after biliary obstruction and cholestasis relief. Therefore, it is not necessary for patients with ECCA to control hypercholesterolemia by taking lipid-lowering medication.

\section{Introduction}

Cholangiocarcinoma (CCA) is the second most common primary hepatobiliary malignancy, contributing to nearly $20 \%$ of hepatobiliary cancer deaths. ${ }^{1}$ CCA is commonly classified into two groups, including intrahepatic CCA (ICCA), which occurs in the bile duct area inside the liver, and extrahepatic CCA (ECCA), which occurs in the bile duct area outside the liver. ${ }^{2}$ ICCA accounts for $10 \%$ to $12 \%$ of liver cancers, whereas ECCA accounts for approximately $1 / 3$ of all biliary tract cancers. ${ }^{3,4}$ The incidence rate of ECCA has decreased in most developed countries, ${ }^{5}$ but recent increases in ECC incidence were observed in China. ${ }^{6}$

The clinical presentation of ICCA is often subtle and may manifest as non-specific symptoms such as weight loss, vomiting, and loss of appetite. ${ }^{7}$ In contrast, ECCA is usually accompanied by jaundice due to biliary tract obstruction. ${ }^{8}$ Increased water-soluble conjugated bilirubin levels can be filtered by the kidney, resulting in darker colored urine. ${ }^{9}$ Moreover, bilirubin does not pass into the duodenum in cases of an obstructed biliary tract, and ECCA patient feces are usually white or white-yellowish of color. ${ }^{10}$ After relieving cholestasis using surgical resection or palliative treatment in patients with ECCA, symptoms including yellow pigmentation of the skin, mucous membranes and sclera, dark urine, and acholic/pale stools may disappear. ${ }^{11,12}$

Cholestasis caused by the bile duct's obstruction is mainly responsible for the signs and symptoms mentioned above. ${ }^{13}$ Moreover, cholestasis may lead to an increase of some laboratory indices such as total bile acid (TBA), alkaline phosphatase (ALP), and gamma-glutamyltranspeptidase (GGT). ${ }^{8}$ Lipid metabolic disorders are also reported in some patients with cholestasis, in whom hypercholesterolemia and abnormal levels of low-density lipoprotein cholesterol (LDLC) are observed. ${ }^{14,15}$ Recently, we found similar lipometabolic disturbances in sporadic cases with ECCA. Of particular concern is whether such patients should be treated with lipid-lowering medication. However, no systematic research has been performed to assess changes in lipid parameters in patients with ECCA.

Therefore, this prospective study was designed to explore serum the change of lipid indices in patients with ECCA and investigate whether removal of biliary obstruction could alleviate the lipid disorder.

\section{Methods}

\section{Participants and Study Design}

We prospectively included consecutive patients admitted to the hepatobiliary surgery department of the Affiliated Hospital of Xuzhou Medical University from October 2019 to May 2020. The patients received a clinical evaluation on admission, including medical history, physical examination, blood tests, and imaging exams. Based on the disease assessment, patients were divided into an ECCA group or a non-ECCA group for further diagnosis. ECCA was defined as a tumor originating in the bile duct outside the liver. Patients with histological confirmation by endoscopic or surgical specimen were finally included in the ECCA group. Patients with hemolytic jaundice, hepatocellular jaundice, pancreatitis, diabetes mellitus, arterial hypertension, and coronary heart disease were excluded. Specific criteria for obstructive jaundice were as follows: (1) clinical signs of icterus (yellow-stained skin, mucosa, and sclera, yellow urine or clay-colored stools), (2) increased serum total bilirubin concentrations (cut-off value $=34.2 \mu \mathrm{mol} / \mathrm{L}$ ), and (3) bile duct dilatation confirmed by imaging examination (ultrasonography, CT or MRCP).

\section{Specimen Collection and Biochemical Tests}

Blood samples were collected from peripheral veins on admission and five days after treatment. After overnight fasting, $5 \mathrm{~mL}$ of blood was collected into serum-separating tubes for liver function and serum lipid detection. An automatic biochemistry analyzer (Roche ${ }^{\circledR}$ Cobas 8000, Roche Diagnostics, Indianapolis, IN, USA) was used to test liver function and measure serum lipid levels using spectrophotometry. Alanine aminotransferase (ALT), 
aspartate aminotransferase (AST), gamma-glutamyl transpeptidase (GGT), alkaline phosphatase (ALP), prealbumin (PA), total protein (TP), albumin (ALB), total bilirubin (TBIL), bilirubin direct (DBIL), total bile acid (TBA), total cholesterol (TC), triglycerides (TG) and cholinesterase (CHE) were detected using commercial kits (Roche Diagnostic, Indianapolis, IN, USA). High-density lipoprotein cholesterol (HDL-C), low-density lipoprotein cholesterol (LDL-C), apolipoprotein A (apoA), apolipoprotein $\mathrm{B}$ (apoB), and lipoprotein (a) (Lp (a)) were tested using commercial kits (Zhicheng Biological Technology Co., Ltd., Shanghai, China). Small and dense low-density lipoprotein cholesterol (sdLDL-C) was measured using a kit produced by Yipunuokang Biotechnology Co., Ltd. (Anhui, China).

Lipoprotein electrophoresis was performed to determine the distribution of lipoprotein components. Serum lipoproteins were stained with Sudan black B (volume ratio 1:1) for $30 \mathrm{~min}$ at room temperature. Mixed samples $(15 \mu \mathrm{L})$ were separated using a $5 \%$ agarose gel and barbitone sodium buffer $(\mathrm{ph}=8.6)$ at $180 \mathrm{~V}$ for $20 \mathrm{~min}$.

\section{Data Collection}

Both personal and clinical data were collected from patients included in the study. Personal data included sex and age. Clinical data included diagnosis, treatment, signs of jaundice, and imaging evidence.

\section{Statistical Analysis}

Statistical analysis was performed using SPSS (version 23.0, SPSS Inc., Chicago, IL, USA). Continuous variables with normal distributions were expressed as mean \pm standard deviation and analyzed using independent sample t-tests, while those with skewed distribution were shown as the median (Q1, Q3) and analyzed using MannWhitney tests or Wilcoxon tests.

\section{Results}

\section{Patient Characteristics}

One hundred and eighty patients met the inclusion criteria and were enrolled in the study. A total of 76 patients were diagnosed with ECCA after histological evaluation. Among these patients, 41 (54.0\%) were diagnosed with proximal cholangiocarcinoma, $9(11.8 \%)$ with middle cholangiocarcinoma, and $26(34.2 \%)$ with distant cholangiocarcinoma. In the ECCA group, 69 patients underwent radical resection, including $1(1.3 \%)$ in stage $0,6(7.9 \%)$ in stage I, $11(14.5 \%)$ in stage II, and $51(67.1 \%)$ in stage III. The other seven patients were in stage IV, and underwent percutaneous transhepatic biliary drainage and stenting for palliative treatment. We enrolled 104 patients in the non-ECCA group. Among these patients, 37 were diagnosed with choledocholithiasis, 3 with cholangitis, 53 with cholecystolithiasis, 9 with cholecystitis, and 2 with cholecystic polypus. In the non-ECCA group, 64 patients underwent cholecystectomy, and 40 underwent common bile duct exploration and T-tube drainage. Significant differences were identified in the number of jaundice cases $(P<0.01)$ between the two groups. There was no significant difference in gender or age between the two groups $(P>0.05)$. Details are shown in Table 1 .

\section{Serum Lipid Levels Differences in the ECCA versus Non-ECCA Groups Before} Treatment

We collected 360 blood samples, of which 180 samples were obtained before treatment and 180 samples after treatment. Before treatment, GGT, ALP, TBIL, DBIL, and TBA levels were significantly higher in the ECCA group than in the non-

Table I Demographics and Clinical Characteristics of the Study Population

\begin{tabular}{|c|c|c|c|}
\hline & $\begin{array}{l}\text { ECCA } \\
(n=76)\end{array}$ & $\begin{array}{l}\text { Non-ECCA } \\
(n=104)\end{array}$ & $P$ value \\
\hline Age (year) & $63.09 \pm|2.3|$ & $59.41 \pm 15.10$ & 0.08 \\
\hline \multicolumn{4}{|l|}{ Sex } \\
\hline Male & 31 (40.8\%) & 57 (59.2\%) & 0.06 \\
\hline \multicolumn{4}{|l|}{ Site } \\
\hline Proximal & $4 \mathrm{I}(54.0 \%)$ & - & \\
\hline Middle & 9 (II.8\%) & - & \\
\hline Distal & $26(34.2 \%)$ & - & \\
\hline \multicolumn{4}{|l|}{ Stage } \\
\hline 0 & I (I.3\%) & - & \\
\hline 1 & $6(7.9 \%)$ & - & \\
\hline II & II (I4.5\%) & - & \\
\hline III & $5 \mathrm{I}(67.1 \%)$ & - & \\
\hline IV & 7 (9.2\%) & & \\
\hline Jaundice & 74 (97.4\%) & 29 (27.9\%) & $<0.01$ \\
\hline $\begin{array}{l}\text { Skin and } \\
\text { mucous }\end{array}$ & 65 (85.5\%) & $23(22.1 \%)$ & $<0.01$ \\
\hline Sclera & 63 (82.9\%) & $22(21.2 \%)$ & $<0.01$ \\
\hline Urine & 62 (8I.6\%) & 28 (26.9\%) & $<0.01$ \\
\hline Stool & 15 (I9.7\%) & I (9.6\%) & $<0.01$ \\
\hline
\end{tabular}

Note: Age is showed as mean \pm standard deviation.

Abbreviation: ECCA, extrahepatic cholangiocarcinoma. 
ECCA group $(P<0.01)$ (Table 2$)$. TC levels were significantly higher in the ECCA group than in the non-ECCA group (5.70 $[4.82,7.40] \mathrm{mmol} / \mathrm{L}$ vs $4.17[3.53,5.06] \mathrm{mmol} / \mathrm{L}, P<0.01)$ (Table 2, Figure 1A). Correspondingly, the ECCA group showed significantly higher levels of sdLDL-C compared with the non-ECCA group $(1.633[1.043,3.072] \mathrm{mmol} / \mathrm{L}$ vs $0.980[0.641,1.329] \mathrm{mmol} / \mathrm{L}, P<0.01)$ (Table 2, Figure 1E). Unexpectedly, the levels of LDL-C were slightly lower in the ECCA group than in the non-ECCA group. $(1.97[0.92,2.84]$ $\mathrm{mmol} / \mathrm{L}$ vs $2.13[1.69,2.85] \mathrm{mmol} / \mathrm{L}, P=0.04$ ) (Table 2 , Figure 1C). Almost all patients in the ECCA group exhibited obstructive jaundice. In the non-ECCA group, 28 patients showed biliary obstruction, including 26 with choledocholithiasis, 2 with cholecystolithiasis, and 1 with cholecystitis. Higher TC levels $(P<0.01)$ and sdLDL-C levels $(P<0.01)$ were observed in the ECCA group than in the biliary obstruction cases in the non-ECCA group (Figure 1B and F). No significant difference was observed in LDL-C levels $(P=$ 0.13 ) between the two groups (Figure 1D). The agreements

Table 2 Liver Function and Serum Lipid Indices of the Patients Before Treatment

\begin{tabular}{|c|c|c|c|}
\hline & $\operatorname{ECCA}(n=76)$ & Non-ECCA $(n=104)$ & $P$ value \\
\hline \multicolumn{4}{|c|}{ Liver function indices } \\
\hline ALT, U/L & $154(85,226)$ & $45(23,120)$ & $<0.01$ \\
\hline AST, U/L & $123(63,189)$ & $34(2 I, 58)$ & $<0.01$ \\
\hline GGT, U/L & $718(333,1068)$ & $70(42,488)$ & $<0.01$ \\
\hline ALP, U/L & $462(288,719)$ & $89(47,264)$ & $<0.01$ \\
\hline $\mathrm{PA}, \mathrm{g} / \mathrm{L}$ & $0.213 \pm 0.077$ & $0.120 \pm 0.065$ & 0.18 \\
\hline TP, g/L & $63.1 \pm 8.3$ & $67.2 \pm 6.0$ & $<0.01$ \\
\hline ALB, $g / L$ & $37.3(34.0,41.9)$ & $39.8(35.6,43.6)$ & 0.02 \\
\hline TBIL, $\mu \mathrm{mol} / \mathrm{L}$ & 227.5 (133.1, 334.5) & $18.8(12.1,36.0)$ & $<0.01$ \\
\hline $\mathrm{DBIL}, \mu \mathrm{mol} / \mathrm{L}$ & 204.1 (II4.2, 275.9) & $8.2(5.5,28.5)$ & $<0.01$ \\
\hline TBA, $\mu \mathrm{mol} / \mathrm{L}$ & $81.2(25.4,174.8)$ & $7.0(4.2,13.5)$ & $<0.01$ \\
\hline CHE, IU/L & 5680 (429I, 706I) & $6771(5626,8087)$ & 0.03 \\
\hline \multicolumn{4}{|c|}{ Serum lipid indices } \\
\hline $\mathrm{TC}, \mathrm{mmol} / \mathrm{L}$ & $5.70(4.82,7.40)$ & $4.17(3.53,5.06)$ & $<0.01$ \\
\hline $\mathrm{TG}, \mathrm{mmol} / \mathrm{L}$ & $2.30(1.72,2.97)$ & $1.46(1.16,2.04)$ & $<0.01$ \\
\hline HDL-C, mmol/L & $0.47(0.20,0.85)$ & $1.00(0.76,1.25)$ & $<0.01$ \\
\hline LDL-C, mmol/L & $1.97(0.92,2.85)$ & $2.13(1.69,2.85)$ & 0.04 \\
\hline sdLDL-C, $\mathrm{mmol} / \mathrm{L}$ & $1.633(1.043,3.072)$ & $0.980(0.64 I, 1.329)$ & $<0.01$ \\
\hline apo Al, g/L & $0.7 \pm 0.5$ & $1.2 \pm 0.4$ & $<0.01$ \\
\hline apo B, g/L & $1.2 \pm 0.4$ & $1.0 \pm 0.3$ & $<0.01$ \\
\hline Lp (a), mg/L & $125(73,171)$ & $159(109,223)$ & $<0.01$ \\
\hline
\end{tabular}

Note: Relatively important indices for the study are shown in bold text.

Abbreviations: ALT, alanine aminotransferase; AST, aspartate aminotransferase; GGT, gamma-glutamyl transpeptidase; ALP, alkaline phosphatase; PA, prealbumin; TP, total protein; ALB, albumin; TBIL, bilirubin total; DBIL, bilirubin direct; TBA, total bile acid; TC, total cholesterol; TG, triglyceride; CHE, cholinesterase; HDL-C high-density lipoprotein cholesterol; LDL-C, low-density lipoprotein cholesterol; sdLDL-C, small and dense low-density lipoprotein cholesterol; apo Al, apolipoprotein Al; apo B, apolipoprotein B; LP (a), lipoprotein (a). between TC and sdLDL-C were good in both groups $(P<0.01)$ (Figure 2A and C). The correlations between TC and LDL-C in the non-ECCA group were strong (Figure 2D), but no significant correlations were observed between $\mathrm{TC}$ and LDL-C in the ECCA group $(P=0.16)$ (Figure 2B).

\section{Serum Lipoprotein Electrophoresis}

Lipoprotein electrophoresis was performed to investigate the reasons for relatively low LDL-C levels in the ECCA group. Lipoproteins in normal serum can mainly be separated into two parts: LDL and HDL particles (Figure 3A$\mathrm{H})$. In the ECCA group, serum samples were detained in the sample wells and lipoproteins failed to separate, suggesting these lipoprotein particles were abnormal (Figure 3A-H). Therefore, cholestasis caused by jaundice may be attributed to the formation of abnormal lipoproteins, which then affect LDL-C detection in biochemistry tests.

\section{Serum Lipid Levels in the ECCA Group After Treatment}

The levels of GGT, ALP, TBIL, DBIL, and TBA dramatically decreased in the ECCA group after treatment $(P<0.01)$ (Table 3 ), suggesting that cholestasis was relieved. Significant differences remained in liver function indices between the ECCA group and the non-ECCA group after treatment $(P<0.01)$ (Table 3). The levels of TC and sdLDL-C significantly decreased after treatment in the ECCA group (Table 3, Figure 4A, C, and E). The median TC levels in the ECCA group before treatment and after treatment were 5.70 (4.82, $7.40)$ and $3.81(2.88,4.57)$, respectively $(P<0.01)$. The median sdLDL-C levels in the two group were 1.633 (1.043, $3.072)$ and $0.830(0.551,1.231)$, respectively $(P<0.01)$. Most patients in the ECCA group showed normal levels of TC and sdLDL-C after treatment, and there were no significant differences in TC and sdLDL-C levels between the ECCA and the non-ECCA groups after treatment (Table 3, Figure 4B, D, and F). Lipoprotein electrophoresis results revealed that patients with ECCA who had serum LDL particles that did not separate showed a normal lipoprotein pattern after treatment (Figure 3A-H).

\section{Discussion}

Serum lipid is the general name of lipids in the blood, mainly including triglycerides (TG), cholesterol (CH), and phospholipids (PL). CH includes free cholesterol and cholesterol esters, which are collectively called total cholesterol (TC). Since TG and TC are hydrophobic substances, 
A

TC

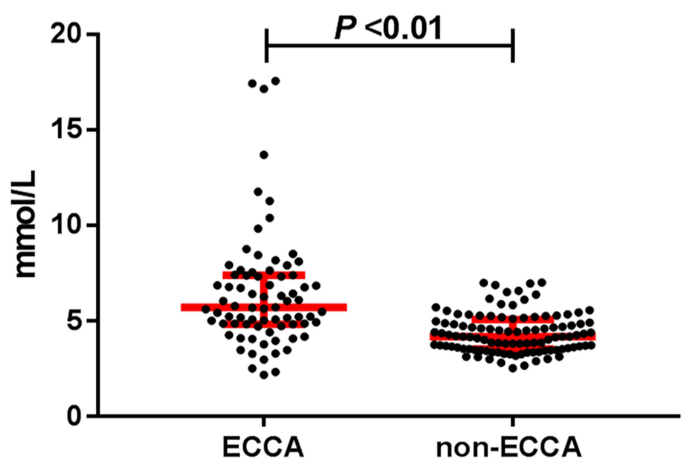

$(n=76)$

C

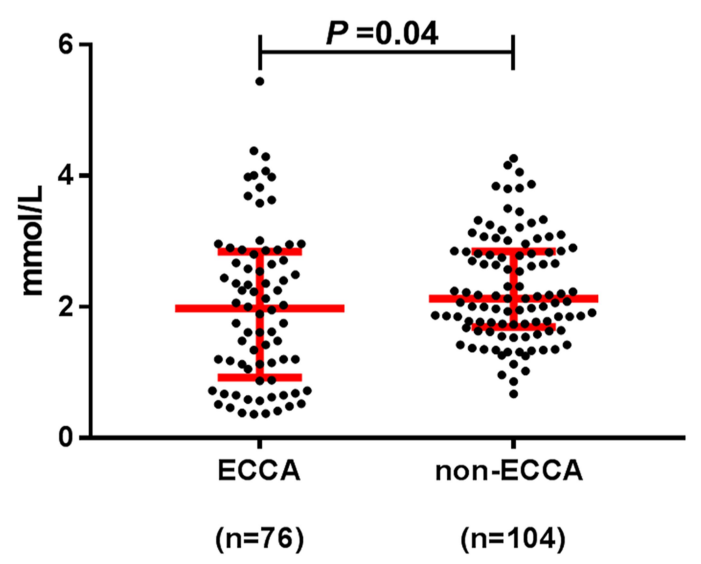

E

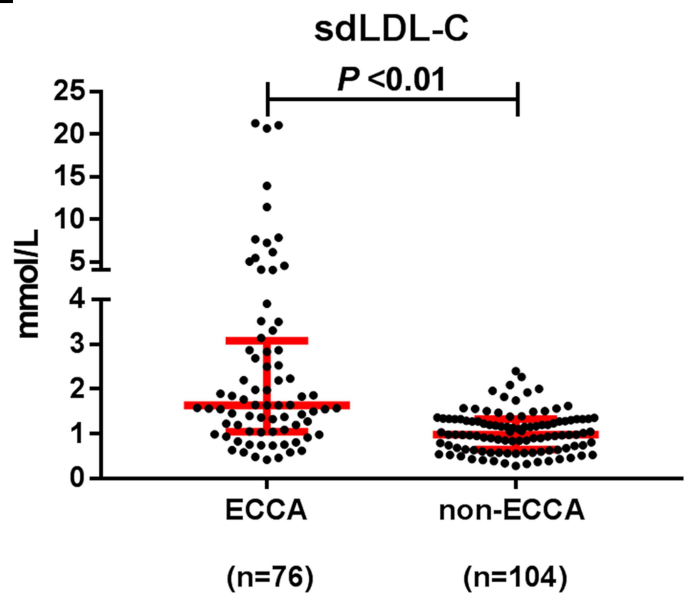

B

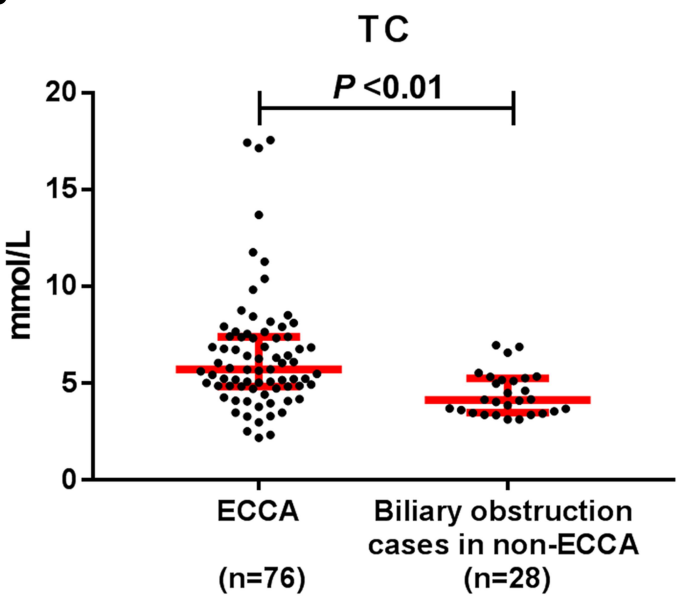

D

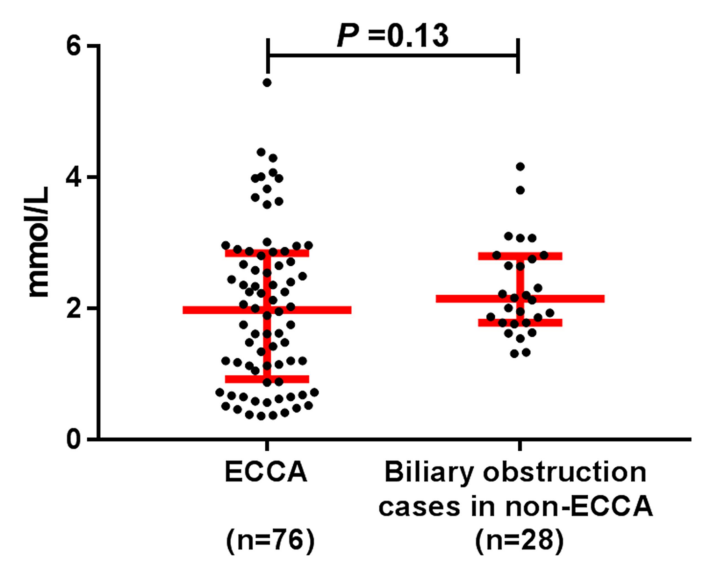

F

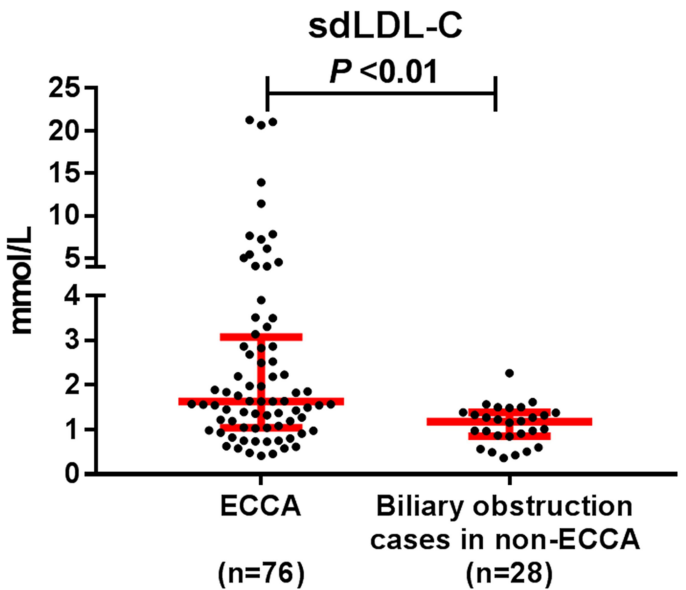

Figure I The levels of TC, LDL-C and sdLDL-C in patients before treatment.

Notes: (A) Comparison of levels of TC between the ECCA group and the non-ECCA group. (B) Comparison of levels of TC between the ECCA group and biliary obstruction cases the non-ECCA group. (C) Comparison of levels of LDL-C between the ECCA group and the non-ECCA group. (D) Comparison of levels of LDL-C between the ECCA group and biliary obstruction cases the non-ECCA group. (E) Comparison of levels of sdLDL-C between the ECCA group and the non-ECCA group. (F) Comparison of levels of sdLDL-C between the ECCA group and biliary obstruction cases the non-ECCA group.

Abbreviations: ECCA, extrahepatic cholangiocarcinoma; TC, total cholesterol; LDL-C, low-density lipoprotein cholesterol; sdLDL-C, small and dense low-density lipoprotein cholesterol. 
A

ECCA

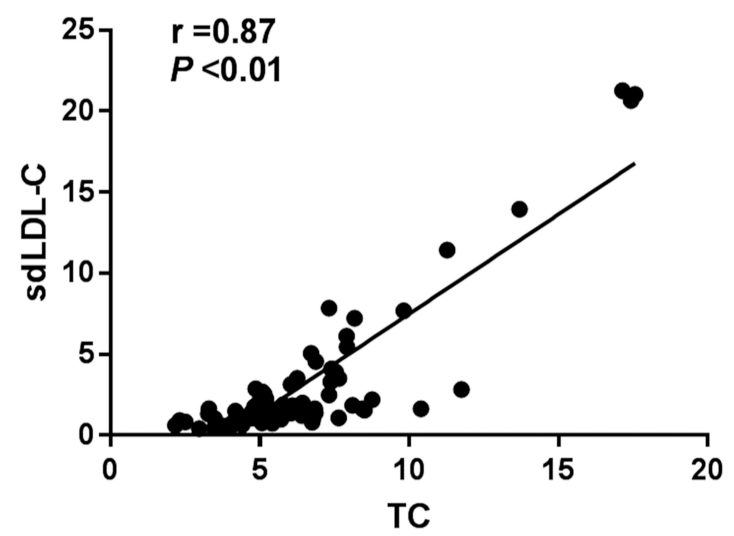

C

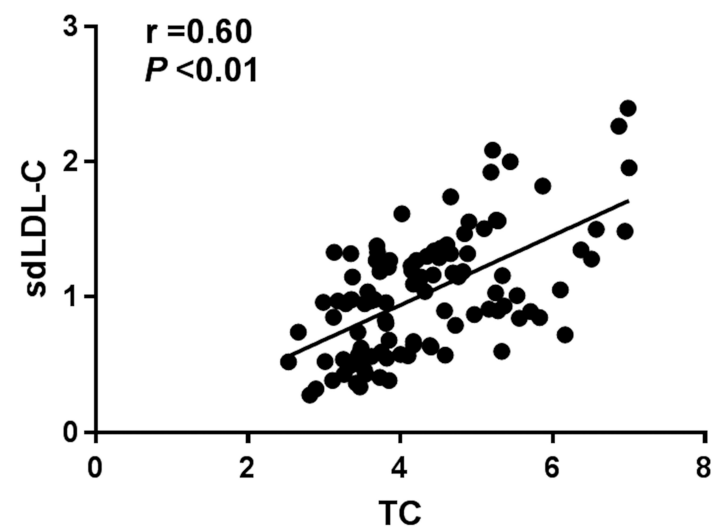

B

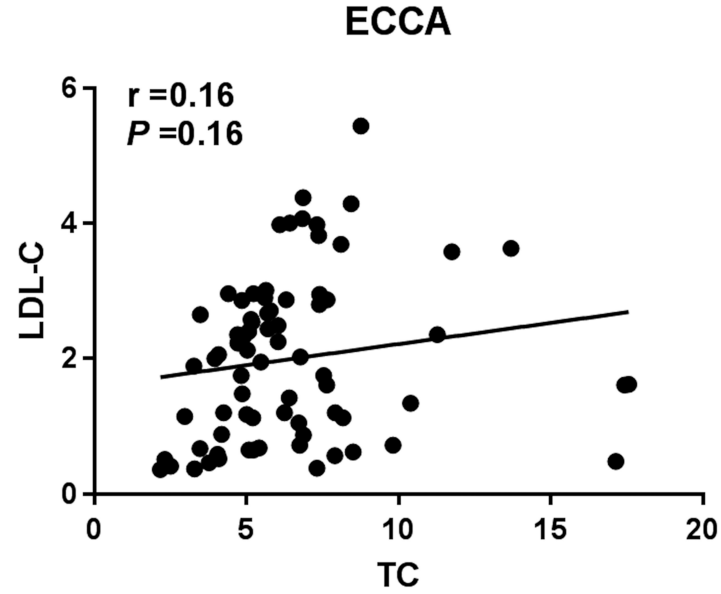

D

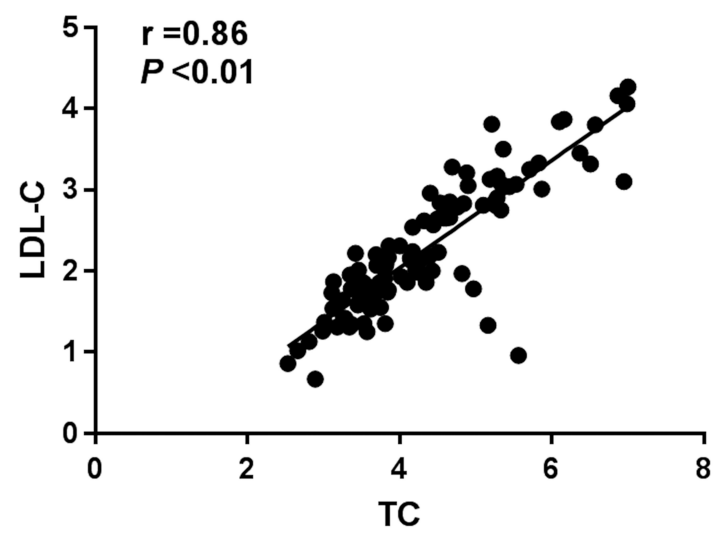

Figure 2 Correlation of serum lipid indices in patients before treatment.

Notes: (A) Correlation of sdLDL-C values and TC values in the ECCA group. (B) Correlation of LDL-C values and TC values in the ECCA group. (C) Correlation of sdLDL-C values and TC values in the non-ECCA group. (D) Correlation of LDL-C values and TC values in the non-ECCA group.

Abbreviations: ECCA, extrahepatic cholangiocarcinoma; TC, total cholesterol; LDL-C, low-density lipoprotein cholesterol; sdLDL-C, small and dense low-density lipoprotein cholesterol.

they must form hydrophilic spherical particles with particular proteins and phospholipids, called lipoproteins, to allow transport in the blood. ${ }^{16}$ Due to the different structures and various compositions, lipoproteins can be divided into chylomicron, very-low-density lipoprotein (VLDL), low-density lipoprotein (LDL), and high-density lipoprotein (HDL) by ultracentrifugation. In agarose gel electrophoresis, it can be separated as chylomicron, $\beta$ lipoprotein, which corresponds to LDL in ultracentrifugation, and $\alpha$-lipoprotein, which corresponds to HDL, from cathode to anode. LDL's primary function is to transport the endogenous cholesterol synthesized by the liver to the extrahepatic tissue. ${ }^{17}$ Therefore, the level of cholesterol in LDL (LDL-C) usually is positively correlated with the level of TC. LDL can be further segmented into many subtypes via ultracentrifugation, such as small and dense LDL (sdLDL). Therefore, the level of sdLDL-C would typically be lower than the level of LDL-C. In this study, we found that patients with ECCA had elevated TC and sdLDL-C levels, and falsely presented with decreased LDL-C levels at admission. We also firstly demonstrated that the TC, sdLDL-C, and LDL-C levels return to normal levels after biliary obstruction removal and cholestasis relief.

Several studies have shown that lipid metabolic disorders are a common complication of cholestasis. ${ }^{14,18}$ Basaranoglu et al reported that patients with intrahepatic cholestasis had a significantly higher TC level than healthy 
A

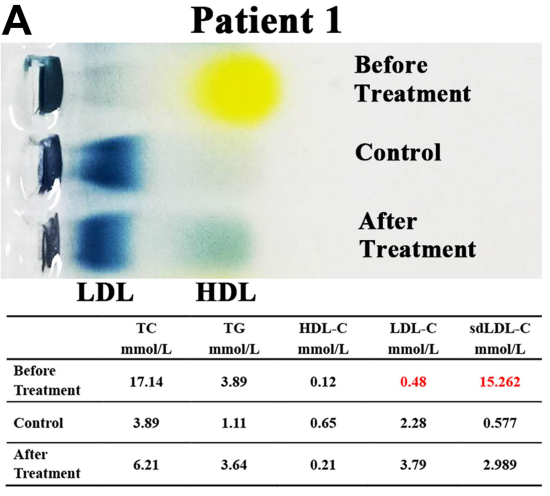

C

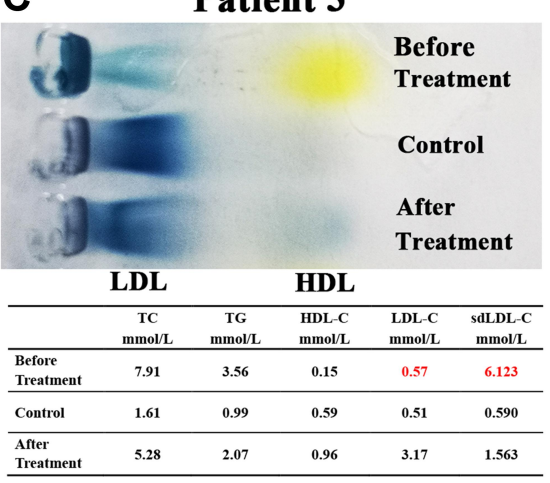

$\mathbf{E}$

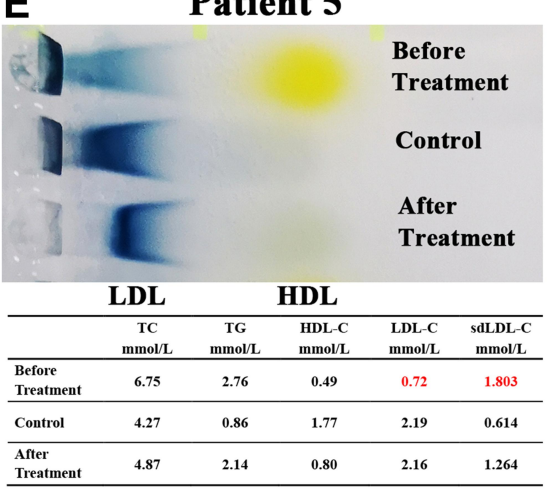

G

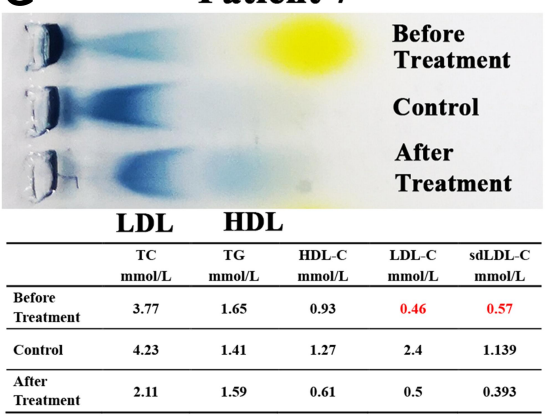

B

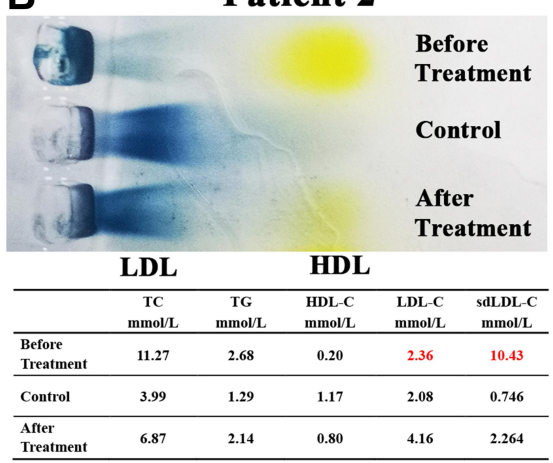

D

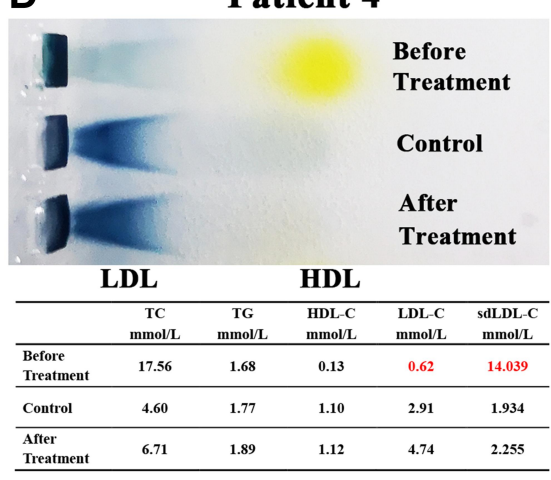

F

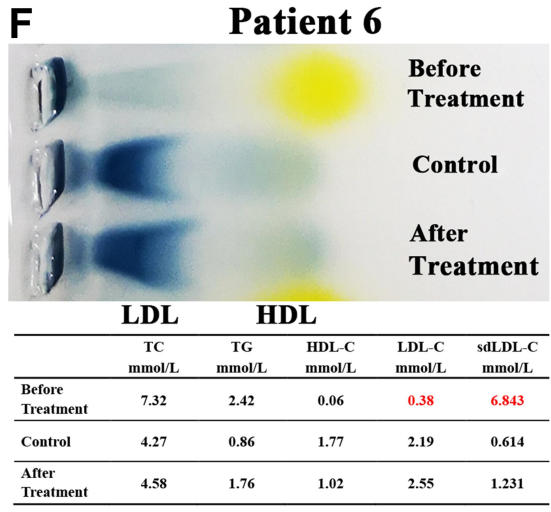

H

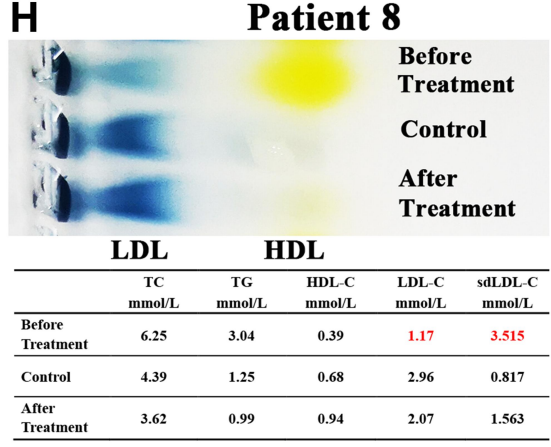

Figure 3 The serum lipoprotein electrophoresis results of 8 ECCA patients who had typically abnormal LDL-C values.

Note: $(\mathbf{A}-\mathbf{H})$ Comparison of serum lipoprotein electrophoresis between non-ECCA patients (control) and ECCA patients before and after treatment. 
Table 3 Liver Function and Serum Lipid Indices of the Patients After Treatment

\begin{tabular}{|c|c|c|c|}
\hline & $\operatorname{ECCA}(n=76)$ & Non-ECCA $(n=104)$ & $P$ value \\
\hline \multicolumn{4}{|c|}{ Liver function indices } \\
\hline ALT, U/L & $47(28,84)$ & $33(20,64)$ & $<0.01$ \\
\hline AST, U/L & $45(24,73)$ & $24(15,38)$ & $<0.01$ \\
\hline GGT, U/L & $168(99,300)$ & $115(53,215)$ & $<0.01$ \\
\hline ALP, U/L & $170(120,289)$ & $98(65,164)$ & $<0.01$ \\
\hline PA, g/L & $0.185 \pm 0.084$ & $0.188 \pm 0.067$ & 0.80 \\
\hline TP, g/L & $59.9 \pm 7.1$ & $65.2 \pm 6.6$ & $<0.01$ \\
\hline ALB, $g / L$ & $34.9(31.3,39.2)$ & $37.8(33.8,41.3)$ & 0.77 \\
\hline TBIL, $\mu \mathrm{mol} / \mathrm{L}$ & $58.5(23.7,135.6)$ & $15.3(10.4,29.3)$ & $<0.01$ \\
\hline $\mathrm{DBIL}, \mu \mathrm{mol} / \mathrm{L}$ & $46.8(15.7,103.4)$ & $6.8(4.5,15.6)$ & $<0.01$ \\
\hline $\mathrm{TBA}, \mu \mathrm{mol} / \mathrm{L}$ & $7.4(3.2,14.1)$ & $3.7(2.4,6.4)$ & $<0.01$ \\
\hline CHE, IU/L & $4505(3676,574 I)$ & $6424(5117,7706)$ & $<0.01$ \\
\hline \multicolumn{4}{|c|}{ Serum lipid indices } \\
\hline $\mathrm{TC}, \mathrm{mmol} / \mathrm{L}$ & $3.81(2.88,4.57)$ & $3.98(3.47,4.48)$ & 0.20 \\
\hline $\mathrm{TG}, \mathrm{mmol} / \mathrm{L}$ & $1.45(1.10,2.10)$ & $1.42(1.15,1.57)$ & 0.15 \\
\hline $\mathrm{HDL}-\mathrm{C}, \mathrm{mmol} / \mathrm{L}$ & $0.76(0.6 \mathrm{I}, 0.95)$ & $0.97(0.75,1.21)$ & $<0.01$ \\
\hline LDL-C, mmol/L & $1.87(1.10,2.44)$ & $1.96(1.57,2.51)$ & 0.12 \\
\hline sdLDL-C, mmol/L & $0.830(0.55 I, I .23 I)$ & $0.980(0.642,1.320)$ & 0.15 \\
\hline apo Al, g/L & $0.7 \pm 0.3$ & $1.2 \pm 0.3$ & $<0.01$ \\
\hline apo B, g/L & $1.0 \pm 0.5$ & $1.0 \pm 0.3$ & 0.31 \\
\hline Lp (a), mg/L & $113(78,200)$ & $165(118,235)$ & $<0.01$ \\
\hline
\end{tabular}

Note: Relatively important indices for the study are shown in bold text. Abbreviations: ALT, alanine aminotransferase; AST, aspartate aminotransferase; GGT, gamma-glutamyl transpeptidase; ALP, alkaline phosphatase; PA, prealbumin; TP, total protein; ALB, albumin; TBIL, bilirubin total; DBIL, bilirubin direct; TBA, total bile acid; TC, total cholesterol; TG, triglyceride; CHE, cholinesterase; HDL-C high-density lipoprotein cholesterol; LDL-C, low-density lipoprotein cholesterol; sdLDL-C, small and dense low-density lipoprotein cholesterol; apo Al, apolipoprotein Al; apo B, apolipoprotein B; Lp (a), lipoprotein (a).

controls $(P<0.01),{ }^{19}$ similar to our results. The TC and sdLDL-C levels were significantly increased in patients with ECCA, who usually show more obvious biliary obstruction and cholestasis. It has also been demonstrated that cholestasis, caused by the injection of sodium taurolithocholate, leads to increased amounts of cholesterol particles in the hepatocytic cytoplasm and the biliary canaliculi of a mouse model. ${ }^{20}$ Compared with patients with acute cholestasis caused by benign diseases, patients with ECCA had higher levels of TC and sdLDL-C, suggesting that hypercholesterolemia is more apparent and severe in chronic cholestasis. Hypercholesterolemia observed in cholestasis is mainly related to the presence of an anomalous lipoprotein named lipoprotein-X (Lp-X). In the obstructive biliary tract, a lipoprotein may reflux into the blood vessels with bile, integrate with TC, apo-C, and esterified cholesterol, to become "mature" $\mathrm{Lp}-\mathrm{X} .^{21}$ This lipoprotein could increase the activity of 3-hydroxy3-methylglutaryl-coenzyme A (HMG-CoA) reductase, and enhance hepatic cholesterol synthesis. ${ }^{18}$
Surprisingly, we found that LDL-C levels were significantly decreased in the ECCA patient group compared with the control group. We noticed that some patients with ECCA showed abnormal LDL-C levels, which may be the reason for the unexpected results. The Friedewald formula $[($ LDL-C) $=$ TC $-($ HDL-C) $-\mathrm{TG} / 2.2 \mathrm{mmol} / \mathrm{L}]$ was used to estimate LDL-C values. ${ }^{22}$ However, the LDL-C values directly quantified by the automatic biochemical analyzer and those calculated according to the Friedewald formula did not match in some patients with ECCA. Another discrepancy was that LDL-C values in these patients were lower than the sdLDL-C values. Since sdLDL is a subtype of LDL, LDL-C values should be greater than sdLDL-C values. Generally, LDL-C is measured using homogeneous methods, where membranes of LDL particles should be firstly dissolved by a specific surfactant, after which cholesterol released from LDL particles can be quantified using coupling reaction of cholesterol esterase, cholesterol oxidase, and peroxidase. ${ }^{15}$ Normal LDL particles should move forward in the electrophoresis, but lipoproteins in patients with ECCA remained in the sampling wells. ${ }^{23}$ These results suggest that physicochemical properties of LDL particles were altered, preventing the surfactant from dissolving the particle membranes, resulting in large amounts of LDL-C failed to be detected. Together with the strong correlation between TC and sdLDL-C, we considered the abnormal LDL-C levels observed in patients with ECCA to be falsely low. Garcia-Hejl et al ${ }^{22}$ also considered a case of low LDL-C in a 69-year-old male with type IIa dyslipidemia as false. That study found a discrepancy between the TC value (9.18 $\mathrm{mmol} / \mathrm{L})$ and the sum of the direct LDL-C $(3.55 \mathrm{mmol} / \mathrm{L})$ and HDL-C values $(0.27 \mathrm{mmol} / \mathrm{L})$. Lp-X present in cholestasis may interfere with homogenous assays for LDL-C measurements, leading to misinterpretation of the lipid status in patients with ECCA. ${ }^{24}$

Fortunately, dyslipidemia in patients with ECCA can be corrected. We detected no significant differences in TC and sdLDL-C observed between the ECCA and the control groups after relieving obstructions. In contrast, indicators of bile duct obstruction (such as GGT and ALP) did not quickly decrease, since GGT and ALP levels may dramatically increase in patients suffering from malignant biliary obstruction. This increase is usually found to be ten times higher than the upper limits of the reference ranges. The abnormal electrophoresis results disappeared after treatment. Other studies have also reported that anomalous LDL-C rapidly decreases after surgical removal of biliary 
A

TC

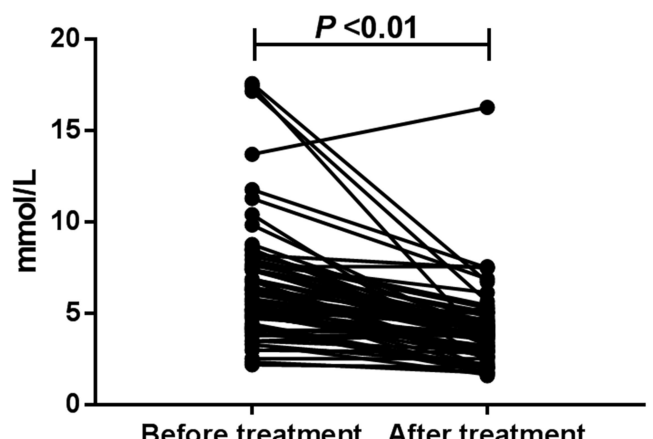

$(n=76)$

$(n=76)$

C

LDL-C

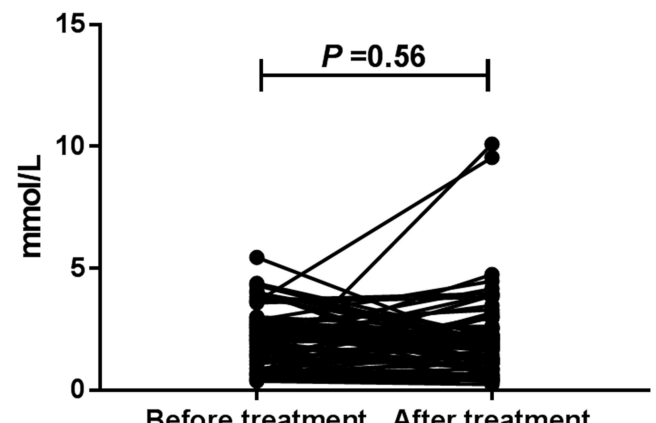

$(n=76)$

$(n=76)$

E

sdLDL-C

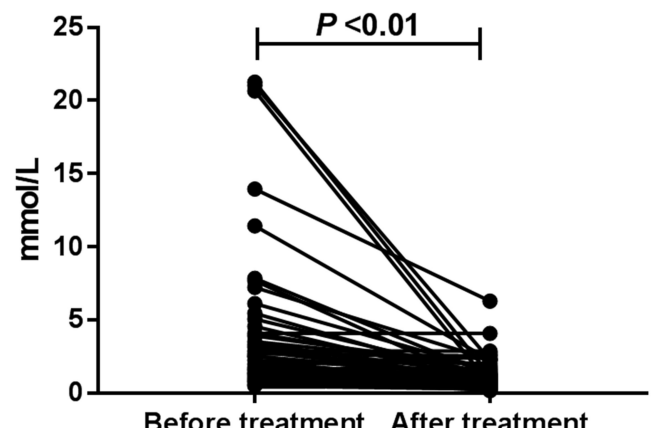

Before treatment After treatment

$(n=76)$

$(n=76)$
B

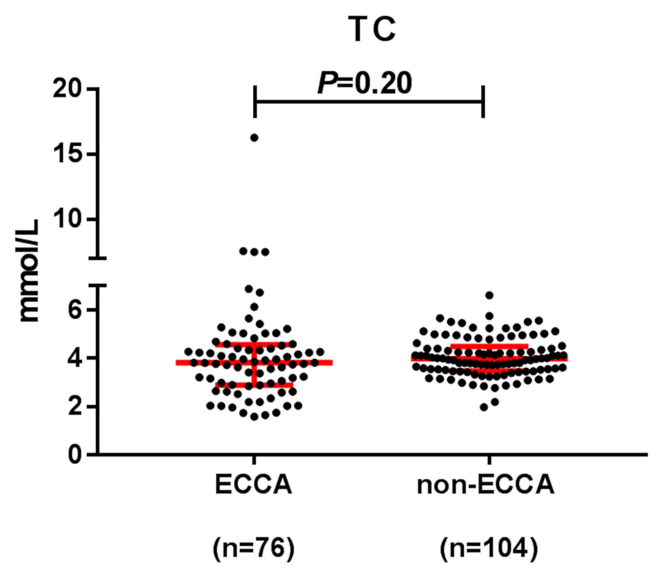

D

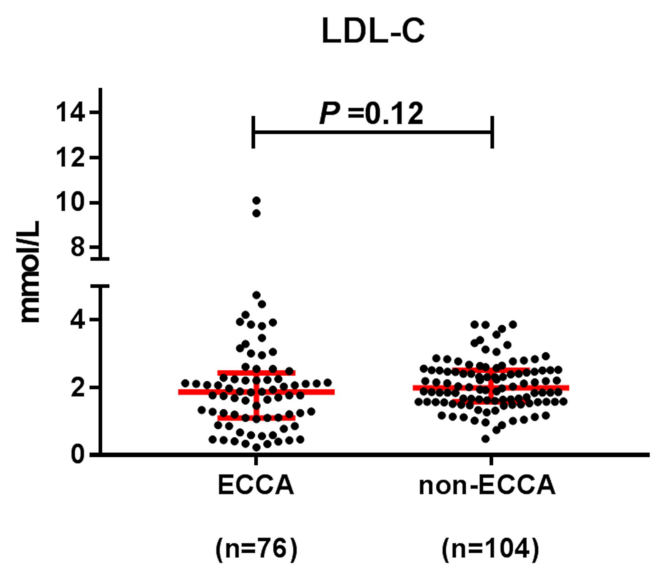

$\mathbf{F}$

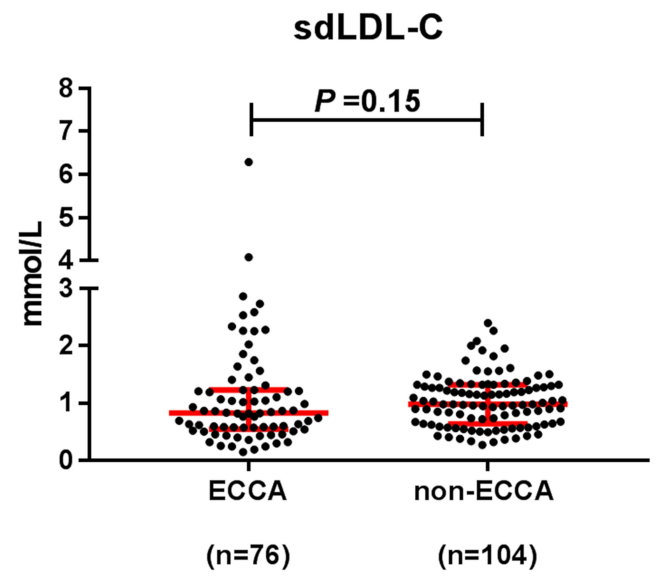

Figure 4 The levels of TC, LDL-C and sdLDL-C in patients after treatment.

Notes: (A) Comparison of levels of TC in the ECCA group between before and after treatment. (B) Comparison of levels of TC between the ECCA group and the nonECCA group after treatment. (C) Comparison of levels of LDL-C in the ECCA group between before and after treatment. (D) Comparison of levels of LDL-C between the ECCA group and the non-ECCA group. (E) Comparison of levels of sdLDL-C in the ECCA group between before and after treatment. (F) Comparison of levels of sdLDL-C between the ECCA group and the non-ECCA group after treatment. 
obstruction, and hypercholesterolemia caused by cholestasis has not been found associated with vascular risk. ${ }^{25,26}$ These results suggest that patients with ECCA do not need to take lipid-lowering medicines, such as statins, for hypercholesterolemia. Therefore, our study holds the potential for avoiding excessive treatments, reducing drug dependence and decreasing medical expenses.

Since Lp-X plays an essential role in the occurrence and development of hypercholesterolemia of cholestasis, the research interests in this area in the next five years should focus on identifying the structures and compositions of the abnormal lipoprotein particle. Based on such data, commercial kits for testing Lp-X can then be developed. The Lp-X test may be useful to infer cholestasis in patients with liver abnormalities of uncertain origin, and may indicate the therapeutic effects and prognosis of patients with malignant biliary obstruction.

Despite our findings and promising prospects, the study did have some limitations. First, the abnormal lipoprotein in patients with ECCA has not been identified in the clinical assay since it could not be separated using electrophoresis. Second, dyslipidemia may not be an exclusive feature for ECCA; other diseases involved in the chronic bile duct obstruction may also present as lipid disorders. Third, the relevance between cholestasis relief and dyslipidemia corrections needs to be further validated with a larger sample size, for this study only included an Asian population.

\section{Conclusion}

ECCA patients showed transiently elevated sdLDL-C and TC levels and falsely presented with low LDL-C results. TC, sdLDL-C, and LDL-C levels were restored to normal levels after biliary obstruction removal and cholestasis relief.

\section{Ethics Approval and Informed Consent}

The study was approved by the Institution Ethics Review Board for Human Studies in the Affiliated Hospital of Xuzhou Medical University. The study was carried out in accordance with the principles of the Declaration of Helsinki. Written informed consent was provided by all participating patients.

\section{Acknowledgments}

We are sincerely grateful to Ping Ma and Shi-Bao Li from the Department of Laboratory Medicine, Affiliated
Hospital of Xuzhou Medical University, who helped us to revise the manuscript.

\section{Author Contributions}

Yi Guo contributed to the study design, and wrote the main manuscript text. Yong-Gen Zhang performed the main experiments, and contributed to statistical analysis. Hong-Chun Li looked up the medical records and conceived the study. YinHai Xu was participate in the conception, communicated with the clinical doctors, collected samples, and revised the manuscript. All of the authors reviewed the manuscript. All authors contributed to data analysis, drafting or revising the article, have agreed on the journal to which the article will be submitted, gave final approval of the version to be published, and agree to be accountable for all aspects of the work.

\section{Funding}

This work was supported by Research Start-up Fund for talent introduction in the Affiliated Hospital of Xuzhou Medical University (Grant No. 2020203002) and the Foundation Research Project (Natural Science Fund) of Jiangsu Province (Grant No. BK20201013).

\section{Disclosure}

The authors report no conflicts of interest in this work.

\section{References}

1. Everhart JE, Ruhl CE. Burden of digestive diseases in the United States Part III: liver, biliary tract, and pancreas. Gastroenterology. 2009;136(4):1134-1144. doi:10.1053/j.gastro.2009.02.038

2. Massa A, Varamo C, Vita F, et al. Evolution of the experimental models of cholangiocarcinoma. Cancers (Basel). 2020;12(8):2308. doi:10.3390/cancers 12082308

3. Altekruse SF, Devesa SS, Dickie LA, McGlynn KA, Kleiner DE. Histological classification of liver and intrahepatic bile duct cancers in SEER registries. J Registry Manag. 2011;38(4):201-205.

4. Van Dyke AL, Shiels MS, Jones GS, et al. Biliary tract cancer incidence and trends in the United States by demographic group, 1999-2013. Cancer. 2019;125(9):1489-1498. doi:10.1002/cncr.31942

5. Bertuccio P, Malvezzi M, Carioli G, et al. Global trends in mortality from intrahepatic and extrahepatic cholangiocarcinoma. $J$ Hepatol. 2019;71(1):104-114. doi:10.1016/j.jhep.2019.03.013

6. Florio AA, Ferlay J, Znaor A, et al. Global trends in intrahepatic and extrahepatic cholangiocarcinoma incidence from 1993 to 2012. Cancer. 2020;126(11):2666-2678. doi:10.1002/cncr.32803

7. Moazzami B, Majidzadeh AK, Dooghaie-Moghadam A, et al. Cholangiocarcinoma: state of the art. J Gastrointest Cancer. 2020;51 (3):774-781. doi:10.1007/s12029-020-00390-3

8. Forner A, Vidili G, Rengo M, Bujanda L, Ponz-Sarvise M, Lamarca A. Clinical presentation, diagnosis and staging of cholangiocarcinoma. Liver Int. 2019;39 Suppl 1:98-107. doi:10.1111/liv.14086

9. Gondal B, Aronsohn A, Systematic A. Approach to patients with jaundice. Semin Intervent Radiol. 2016;33(4):253-258. doi:10.1055/ s-0036-1592331 
10. Sullivan JI, Rockey DC. Diagnosis and evaluation of hyperbilirubinemia. Curr Opin Gastroenterol. 2017;33(3):164-170. doi:10.1097/MOG.0000000000000354

11. Abu-Hamda EM, Baron TH. Endoscopic management of cholangiocarcinoma. Semin Liver Dis. 2004;24(2):165-175. doi:10.1055/s-2004-828893

12. Esnaola NF, Meyer JE, Karachristos A, Maranki JL, Camp ER, Denlinger CS. Evaluation and management of intrahepatic and extrahepatic cholangiocarcinoma. Cancer. 2016;122(9):1349-1369.

13. Rizzo A, Ricci AD, Frega G, et al. How to choose between percutaneous transhepatic and endoscopic biliary drainage in malignant obstructive jaundice: an updated systematic review and meta-analysis. In Vivo (Brooklyn). 2020;34(4):1701-1714. doi:10.21873/invivo.11964

14. Fellin R, Manzato E. Lipoprotein-X fifty years after its original discovery. Nutr Metab Cardiovasc Dis. 2019;29(1):4-8. doi:10.1016/j.numecd.2018.09.006

15. Kurosawa H, Yoshida H, Yanai H, Ogura Y, Hirowatari Y, Tada N. Comparative study between anion-exchange HPLC and homogeneous assay methods in regard to the accuracy of high- and low-density lipoprotein cholesterol measurement. Clin Biochem. 2007;40(16-17):1291-1296. doi:10.1016/j.clinbiochem.2007.07.008

16. Feingold KR, Grunfeld C. Introduction to Lipids and Lipoproteins. In: Feingold KR, Anawalt B, Boyce A, editors. Endotext. South Dartmouth (MA): MDText.com, Inc. 2000; 22-33.

17. Beisiegel U. Lipoprotein metabolism. Eur Heart J. 1998;19 Suppl A: A20-A23.

18. Nuno-Lambarri N, Barbero-Becerra VJ, Uribe M, Chavez-Tapia NC. Elevated cholesterol levels have a poor prognosis in a cholestasis scenario. J Biochem Mol Toxicol. 2017;31(1):1-6. doi:10.1002/ jbt. 21831
19. Basaranoglu S, Agacayak E, Ucmak F, et al. The role of vitamin B1-B2 and plasma lipid profile in intrahepatic cholestasis of pregnancy. J Perinat Med. 2017;45(4):461-465. doi:10.1515/jpm2015-0337

20. Bonvicini F, Gautier A, Gardiol D, Borel GA. Cholesterol in acute cholestasis induced by taurolithocholic acid. A cytochemical study in transmission and scanning electron microscopy. Lab Invest. 1978;38 (4):487-495.

21. Manzato E, Fellin R, Baggio G, Walch S, Neubeck W, Seidel D. Formation of lipoprotein-X. Its relationship to bile compounds. J Clin Invest. 1976;57(5):1248-1260. doi:10.1172/JCI108393

22. Garcia-Hej1 C, Vest P, Renard C, Merens-Gonthier A, Boukhira A, Thefenne-Astier H. Falsely low LDL cholesterol results and cholestasis. Clin Chem. 2006;52(11):2125-2127. doi:10.1373/ clinchem.2006.072249

23. Kattah L, Gomez A, Gutierrez S, et al. Hypercholesterolemia due to Lipoprotein X: case report and thematic review. Clin Med Insights Endocrinol Diabetes. 2019;12:1179551419878687. doi:10.1177/ 1179551419878687

24. Heimerl S, Boettcher A, Kaul H, Liebisch G. Lipid profiling of lipoprotein X: implications for dyslipidemia in cholestasis. Biochim Biophys Acta. 2016;1861(8 Pt A):681-687. doi:10.1016/j. bbalip.2016.04.016

25. Frohlich J. Role of lecithin: cholesterol acyltransferase and apolipoprotein A-I in cholesterol esterification in lipoprotein-X in vitro. $J$ Lipid Res. 1995;36(11):2344-2354. doi:10.1016/S0022-2275(20) 39715-7

26. Allocca M, Crosignani A, Gritti A, et al. Hypercholesterolaemia is not associated with early atherosclerotic lesions in primary biliary cirrhosis. Gut. 2006;55(12):1795-1800. doi:10.1136/gut.2005.079814
OncoTargets and Therapy

\section{Publish your work in this journal}

OncoTargets and Therapy is an international, peer-reviewed, open access journal focusing on the pathological basis of all cancers, potential targets for therapy and treatment protocols employed to improve the management of cancer patients. The journal also focuses on the impact of management programs and new therapeutic agents and protocols on patient perspectives such as quality of life, adherence and satisfaction. The manuscript management system is completely online and includes a very quick and fair peer-review system, which is all easy to use. Visit http://www.dovepress.com/ testimonials.php to read real quotes from published authors. 